\title{
A Development of Graphical Interface for Decision Making Process Including Real-Time Consistency Evaluation
}

\author{
Joong-Ho Lee, Ki-Won Yeom, and Ji-Hyung Park* \\ Intelligence Interaction Research Center, Korea Institute of Science and Technology, \\ HaWolGok 39-1, Seoul, 130-650, Korea \\ jhpark@kist.re.kr
}

\begin{abstract}
Decision making problems are often imprecise and changeable because of potential inconsistency in human thinking. Although AHP gives a desirable guide to the reasonable solution via consistency ratio, there is still possibility of containing inconsistence during process. Therefore, an important step in many applications of decision making problems is to perform a consistency analysis in real-time. We introduce a new method of priority setting in decision making processes, which is implemented as an interactive and convenient graphical interface of the decision making problem. It is designed to support the real-time consistency evaluation. The conventional AHP does not provide graphical user interface and is impossible to monitor the interim findings in the middle of process, and is difficult to predict the difference of results when changing pair-wise comparison conditions, and is difficult to monitor the consistency of human judgment during operation. The proposed real-time calculation algorithm and visualization method is developed to realize effective and reliable decision making environment, and is verified its merit through the exemplary case. In addition, we propose new algorithm of evaluating consistency level. The rationality tension is proposed as a new index for evaluating a real-time consistency analysis with interactive graphical user interface. It is desirable for a system to provide fast and visible information of consistency in decision making processes.
\end{abstract}

Keywords: Decision Making, Priority Setting, AHP, Visualization, Interactive process, Consistency Ratio.

\section{Introduction}

The analytic hierarchy process, AHP [1] is a comprehensive, logical and structured framework for solving a priority setting. It improves understanding of complex decisions by decomposing the problem in a hierarchical structure. This method incorporates all relevant decision criteria and allows the decision maker to determine the trade-offs among alternatives by pair-wise comparison.

\footnotetext{
* Corresponding author.
} 


\subsection{Decomposition of the Decision Problem}

At first, User defines the problem. Fig 1 shows the exemplary hierarchy of the problem. To acquire the best choice among alternatives, user should define decision criterions. In other words, the problem is which alternative cloud be the best choice to meet the goal considering all criterions.

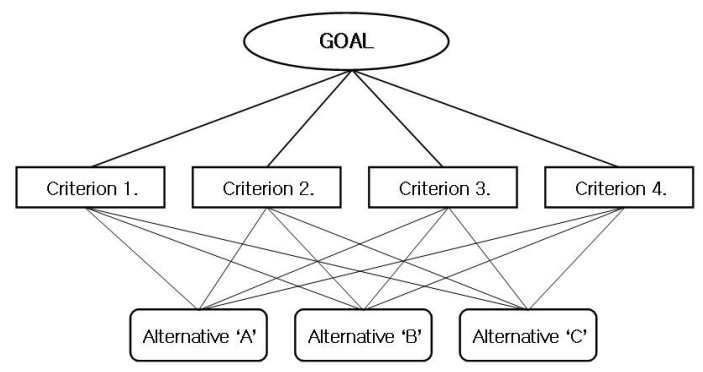

Fig. 1. To find the best choice for a desirable GOAL, user defines criterions and derives priority setting considering each criterion. The above shows exemplary decomposition of four criterions and three alternatives.

\subsection{Pair-Wise Comparison Between Alternatives}

AHP gives an effective evaluation methodology - pair-wise comparison, which guides users to decide priorities of alternatives by considering only two alternatives at one time. Fig. 2 shows exemplary AHP decision table. All pair-wise comparison values are filled up on the table. This table is called comparison matrix (M). Usually, it is recommended to use $1,3,5,7,9,1 / 3,1 / 5,1 / 7,1 / 9$ for the value.

* Criterion 1's comparison table.

\begin{tabular}{|l|c|c|c|}
\hline & Alternative 'A' & Alternative 'B' & Alternative 'C' \\
\hline Alternative 'A' & 1 & 3 & $1 / 5$ \\
\hline Alternative 'B' & $1 / 3$ & 1 & $1 / 7$ \\
\hline Alternative 'C' & 5 & 7 & 1 \\
\hline
\end{tabular}

$$
\mathbf{M}=\left(\begin{array}{ccc}
1 & 3 & 1 / 5 \\
1 / 3 & 1 & 1 / 7 \\
5 & 7 & 1
\end{array}\right)
$$

Fig. 2. The pair-wise comparisons are filled up on the table. This table is called comparison matrix $\mathrm{M}$.

\subsection{Synthesis of the Priorities}

Synthesizing the comparisons is to get the priority setting of the alternatives with respect to each criterion and the weights of each criterion with respect to the goal. User calculates eigen values or geometric averages for each alternatives. Local priorities are then multiplied by the weights of the respective criterion. Finally, the results are summed up to get the overall priority of each alternative. The local priority setting of the above table in Fig. 2 is shown on table 1. 
Table 1. The local priority setting of criteron 1

\begin{tabular}{|c|c|c|c|}
\hline & Alternative 'A' & Alternative 'B' & Alternative 'C' \\
\hline Weight & 1.182 & 2.759 & 0.306 \\
\hline percentage & $27.8 \%$ & $65 \%$ & $7.2 \%$ \\
\hline Rank & 2 & 1 & 3 \\
\hline
\end{tabular}

\section{Consistency Evaluation in AHP}

The consistency index C.I is used for evaluating rationality of the AHP priority setting result. If C.I is too large the result is regarded to be unreasonable because there may be too much antinomy in comparison. C.I is an important index to verify the consistence of decision making process.

\subsection{Consistency Index}

As a result above in Fig. 2, Alternative ' $B$ ' is the best decision( $65 \%$ of priority). Since ' $B$ '> 'A' and 'A'> 'C', logically, we hope that 'B'> $C$ '. This logic of preference is called transitive property. If user's judgment of comparison ' $\mathrm{B}$ ' vs ' $\mathrm{C}$ ' is transitive, then this decision process can be considered as consistent one. On the contrary, if the user judged ' $\mathrm{C}$ ' is more important than ' $\mathrm{B}$ ', this decision has inconsistent judgment. To evaluate the decision's consistency, a numerical index is used, which is closely related to the transitive property. A comparison matrix M(as shown on Fig. 2) is said to be consistent if,

$$
\mathbf{m}_{\mathrm{ij}} \times \mathbf{m}_{\mathrm{jk}}=\mathbf{m}_{\mathrm{ik}} \text { for all } \mathrm{i}, \mathrm{j}, \mathrm{k}
$$

For consistent reciprocal matrix, the largest eigen value is equal to the number of alternatives, or $\lambda_{\max }=\mathrm{n}$. Then a measure of consistency is below,

$$
\begin{gathered}
\mathbf{C I}=\left(\boldsymbol{\lambda}_{\max }-\mathbf{n}\right) /(\mathbf{n}-\mathbf{1}) \quad \text { where } \lambda: \text { eigen value of matrix } M \\
\mathrm{n}: \text { number of alternatives }
\end{gathered}
$$

Thus in our previous example shown on Fig. 2, we have $\lambda_{\max }=3.054$ and three comparisons, or $\mathrm{n}=3$, thus the consistency index is 0.027 .

\subsection{Consistency Ratio}

The consistency level is measured by comparing it with the appropriate one. The appropriate consistency index is called random consistency index (R.I). It is randomly generated reciprocal matrix using scale $1 / 9,1 / 8, \ldots 1, \ldots 8,9$. The random consistency index is shown on the Table. 2.

Table 2. The random consistency index R.I with respect to size of matrix M

\begin{tabular}{|c|c|c|c|c|c|c|c|c|c|c|}
\hline $\mathrm{n}$ & 1 & 2 & 3 & 4 & 5 & 6 & 7 & 8 & 9 & 10 \\
\hline $\mathrm{RI}$ & 0 & 0 & 0.58 & 0.9 & 1.12 & 1.24 & 1.32 & 1.41 & 1.45 & 1.49 \\
\hline
\end{tabular}

The consistency ratio(C.R) is a comparison between consistency index(C.I) and random consistency index(R.I), or in formula, 


\section{C.R = C.I / R.I}

If the value of consistency ratio is small enough or equal to $10 \%$, the inconsistency is acceptable. If the consistency ratio is greater than $10 \%$, we need to revise the subjective judgment. For our previous example, we have C.I=0.027 and R.I for $n=3$ is 0.58 , then we have, $4.66 \%$ for C.R. Thus, this subjective judgment can be considered as consistent process.

\section{Real-Time Priority Setting}

The real-time priority setting that we introduce in this paper is a new method of decision making problem. It is composed of a real-time calculation algorithm and visualization. As the computer environment growing fast, a study for interactive operation between human and computer for decision making problem is newly issued. The real-time priority setting is suitable for up-to-date computing environment to complement defects of AHP. The real-time priority setting algorithm replaces stepwise manual calculation process of AHP. This algorithm can refresh interim finding immediately after the change of pair-wise comparisons. An interim finding is shown on display with graphical symbols which increase visual understanding. User can sense current situations directly via graphical expressions. And when the conditions changed, for example, new emergence of additional alternative, user can confirm the updated result only by handling a few graphical factors instead of remaking new AHP table and restarting the process from the beginning.

\subsection{Graphical Expression}

A conventional AHP shows the result only by numerical table, which makes user hard to understand the result instantly. It is certain that more legible information with graphical expression improve the efficiency significantly. The Real-Time Graphical Expression gives not only numerical information but also symbolic expression such as circle, line, color, size and thickness. It gives information of current status for users to understand interim findings rapidly and easily. Fig. 3 shows the exemplary symbolic expression of the real-time priority setting.

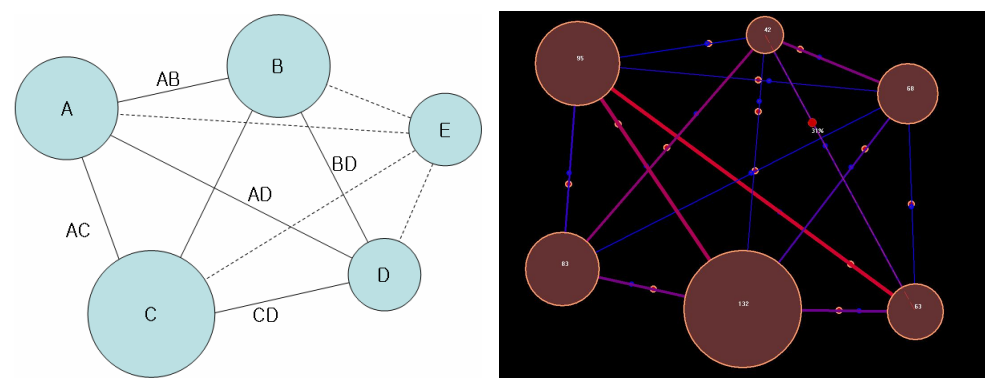

Fig. 3. The real-time priority setting method uses graphical expression as shown. Using circles, lines and their sizes, colors, the interim finding can be monitored interactively. 
Circles represent alternatives. They have basic information of alternative's features, remark, keyword, weight percentage. Each size of the circles represents its current weight. As a pair-wise comparison judged, a line is created by human input gesture. The line represents comparison value and its consistency level. During decision, users may create many notes. The system allows users to edit remarks on the circle.

\subsection{Algorithm}

The real-time priority setting method derives the weight of alternatives via iterative linear algebra. This method uses the algorithm which result is proved to be nearly corresponds to conventional AHP method. A case shows prioritizing among 3 alternatives (A, B, C). When a pair-wise comparison is decided, an interim finding is updated immediately as below.

$$
\mathrm{P}_{\mathrm{A}}^{\mathrm{n}}=\left(\mathrm{P}^{\mathrm{n}-1}{ }_{\mathrm{A}}+\mathrm{R}_{\mathrm{AB}} \times\left(\mathrm{P}^{\mathrm{n}-1}{ }_{B}+\mathrm{P}^{\mathrm{n}-1}{ }_{A}\right)+\mathrm{R}_{\mathrm{AC}} \times\left(\mathrm{P}^{\mathrm{n}-1}{ }_{C}+\mathrm{P}^{\mathrm{n}-1}{ }_{A}\right)\right) / \mathrm{N}
$$

Where, $\mathrm{P}_{\mathrm{A}}^{\mathrm{n}}$ is the weight of alternative ' $\mathrm{A}$ ' in $\mathrm{n}$ 's iteration, $\mathrm{R}_{\mathrm{AB}}$ is the priority value between ' $A$ ' and ' $\mathrm{B}$ '. $\mathrm{N}$ is the number of terms of algebra. As the iteration repeated (if $\mathrm{n}$ is sufficiently large), a difference between $\mathrm{P}^{\mathrm{n}}{ }_{\text {all }}$ and $\mathrm{P}^{\mathrm{n}-1}$ all comes to decrease and $\mathrm{P}^{\mathrm{n}}$ all converges to the unique value.

$$
\mathrm{P}_{\text {all }}^{\mathrm{n}}-\mathrm{P}_{\text {all }}^{\mathrm{n}-1}<\mathrm{Z}
$$

Iteration halt constant $\mathrm{Z}$ reduces the number of iteration. If $\mathrm{Z}$ is sufficiently small, final $\mathrm{P}^{\mathrm{n}}{ }_{\text {all }}$ corresponds to the unique value. Table 2 shows the result of real-time priority setting for the same comparison matrix $M$ in Fig. 2. Furthermore since this calculation can be done even though not all pair-wise comparisons decided, user can calculate interim findings. For example, if $\mathrm{R}_{\mathrm{AC}}$ is not decided yet, it is set to zero. The result could express reasonable interim finding while the conventional AHP can not show any reasonable index in the middle of the process.

\section{Rationality Tension}

The consistence index C.I is used for evaluating rationality of the AHP result. If C.I is too large the result is regarded to be unreasonable because there may be too much antinomy in comparison decision. In this paper, new index called Rationality Tension (R.T) is introduced for evaluating the consistency of the result.

\subsection{Algorithm}

Since a human thinking has a possibility of containing inconsistency along to the decision making, rapid interaction for informing that he/she is going with too much inconsistency could provide considerable advantage. When the pair-wise comparison is changed, $\mathrm{K}$ value is updated as below.

$$
\mathrm{K}_{\mathrm{AB}}=\left|\mathrm{R}_{\mathrm{AB}}-\mathrm{P}_{\mathrm{A}}^{\mathrm{n}} / \mathrm{P}_{\mathrm{B}}^{\mathrm{n}}\right|
$$

$\mathrm{K}_{\mathrm{AB}}$ is an index of difference between current pair-wise comparison between ' $\mathrm{A}$ ' and ' $\mathrm{B}$ '. On condition that iteration of real-time calculation is enough to acquire the 
valid value, a priority is stable on balance of itself and other's effects. If all $\mathrm{K}$ values of alternative 'A' are small enough for all other's relations, there are small antinomies with respect to the alternative ' $A$ '. While AHP considers only one representative value for a matrix $\mathrm{M}$, this algorithm makes it possible to consider all relation's consistency levels case by case.

$$
\text { R.T }=\operatorname{Max}\left\{\mathrm{K}_{\mathrm{AB}}, \mathrm{K}_{\mathrm{AC}}, \mathrm{K}_{\mathrm{BC}}, \ldots\right\}
$$

R.T is 'rationality tension' which is proposed in this paper for a new index of consistency level. The rationality tension R.T corresponds to C.I. It can be calculated at any stage of process. User can monitor the R.T to check the validity of current decision whenever he/she needs to inspect whether the relation keeps too much contradictions or not. A Monitoring of R.T is available on display with various color and thickness of line. As the R.T increases large, color and thickness are changed to be detected clearly by user. User can modify the pair-wise comparison judgment which includes high R.T. This advantage leads users to qualify their conclusion more effectively.

\subsection{Visualization}

If a comparison line has low R.T, it is expressed as the thin and dark colored. On the other hand, a comparison line which has high R.T is expressed thick with deep red color as shown on Fig. 4.

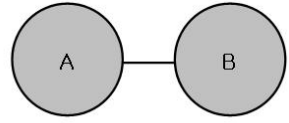

(a) Low RT

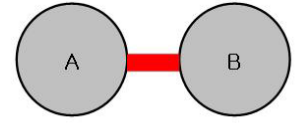

(a) High RT

Fig. 4. The Real-Time Graphical Expression shows the current consistency information R.T with thickness and color of line for user to understand instantly.

When user operates decision making with pair-wise comparison, the real-time priority setting gives information about R.T instantly. It would help users to find where the inconsistency occurred significantly during decision. And it leads users to modify comparisons to the reasonable consistency level.

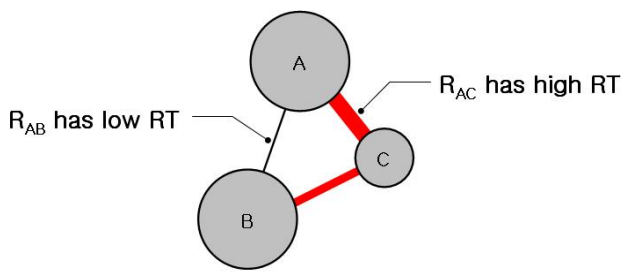

Fig. 5. Real-time expression of consistency levels helps user to derive reasonable result in decision making 


\subsection{C.R(Consistency Ratio) vs R.T(Rationality Tension)}

According to our study, C.R $=10 \%$ corresponds to $\mathrm{R} . \mathrm{T}=10$ approximately. If $\mathrm{R} . \mathrm{T}$ is increased up to 10 , the depth of line becomes thick and color becomes red, which means that user should consider previous comparisons again. Fig 6 and Table 3 shows the results done by real-time priority setting of the matrix M in Fig. 2.

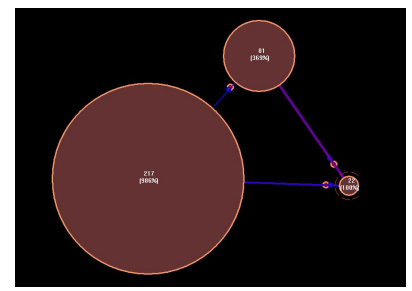

Fig. 6. The real-time priority setting result of the matrix $M$ in Fig 2

Table 3. The real-time priority setting shows approximately same result to that of AHP

\begin{tabular}{|c|c|c|c|c|c|c|}
\hline $\mathrm{P}_{\mathrm{A}}$ & $\mathrm{P}_{\mathrm{B}}$ & $\mathrm{P}_{\mathrm{C}}$ & $\mathrm{K}_{\mathrm{AB}}$ & $\mathrm{K}_{\mathrm{AC}}$ & $\mathrm{K}_{\mathrm{BC}}$ & R.T \\
\hline $25.3 \%$ & $65 \%$ & $7.2 \%$ & 2.2 & 4.4 & 2.8 & 4.4 \\
\hline
\end{tabular}
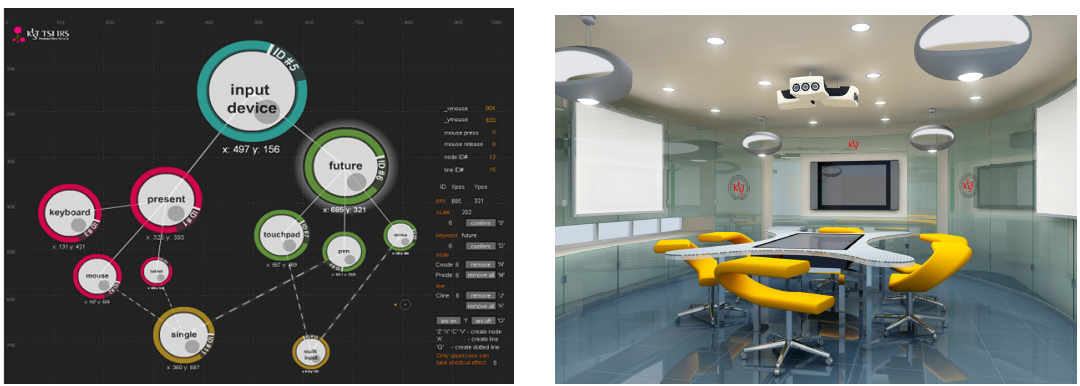

Fig. 7. The Graphical Interface for Decision Making System has installed in IRS as an interactive solution for supporting the priority setting

\section{Conclusion}

The real-time priority setting enables users to monitor interim findings with interactive user interface. Symbolic expression improves visual understandings. And when defining pair-wise conditions, user is able to evaluate whether the decision retain the desirable consistency. It is more effective and fast than conventional AHP process. Finally this method proved to be suitable for remote, collaborative, large displayed environment by sharing visual symbolic information among users. And it provides users with real-time responses relative to users operation. This method has been substantially adopted into IRS(Intelligent Responsive Space) digital workbench 
which is being developed as a Tangible Space Initiative project at Intelligence Interaction Research Center in KIST(Korea Institute of Science and Technology).

\section{References}

1. Saaty, T.L.: The Analytic Hierarchy Process: Planning, Priority Setting, Resource Allocation, pp. 56-57. McGraw-Hill, NewYork (1980)

2. Saaty, T.L.: Decision Making, Scaling, and Number Crunching. Decision Sciences, pp. 404-409 (1989)

3. Park, J.-H., Lee, J.-H., Yeom, K.-W., Lee, S.-S., Eom, J.-I.: Graphical Expression Method for Decision Process Support, HCI2006 (2006)

4. James, S.D.: Remarks on the Analytical Hierarchy Process, Management Science, pp. 249 $258(1990)$

5. Saaty, T.L., Jo, G.-T., Hong, S.-W., Gouen, C.-S.: Decision Making for Leaders, RWS pub. (1995)

6. Escobar, M.T., aguaron, J., Jimenez, J.M.M.: A note on AHP group consistency for the row geometric mean priorizatio, European Journal of Operational Research

7. Escobar, M.T., aguaron, J., Jimenez, J.M.M.: Consistency stability intervals for a judgment in AHP decision support, European Journal of Operational Research 